ARTICLE

\title{
Observation of a shape resonance of the positronium negative ion
}

Koji Michishio', Tsuneto Kanai ${ }^{2}$, Susumu Kuma², Toshiyuki Azuma², Ken Wada³, Izumi Mochizuki ${ }^{3}$, Toshio Hyodo ${ }^{3}$, Akira Yagishita ${ }^{3} \&$ Yasuyuki Nagashima ${ }^{1}$

When an electron binds to its anti-matter counterpart, the positron, it forms the exotic atom positronium (Ps). Ps can further bind to another electron to form the positronium negative ion, $\mathrm{Ps}^{-}\left(\mathrm{e}^{-} \mathrm{e}^{+} \mathrm{e}^{-}\right)$. Since its constituents are solely point-like particles with the same mass, this system provides an excellent testing ground for the three-body problem in quantum mechanics. While theoretical works on its energy level and dynamics have been performed extensively, experimental investigations of its characteristics have been hampered by the weak ion yield and short annihilation lifetime. Here we report on the laser spectroscopy study of $\mathrm{Ps}^{-}{ }^{-}$, using a source of efficiently produced ions, generated from the bombardment of slow positrons onto a Na-coated W surface. A strong shape resonance of ${ }^{1} \mathrm{P}^{\circ}$ symmetry has been observed near the Ps $(n=2)$ formation threshold. The resonance energy and width measured are in good agreement with the result of three-body calculations.

\footnotetext{
${ }^{1}$ Department of Physics, Tokyo University of Science, 1-3 Kagurazaka, Shinjuku, Tokyo 162-8601, Japan. ${ }^{2}$ Atomic, Molecular and Optical Physics Laboratory, RIKEN, 2-1 Hirosawa, Wako, Saitama 351-0198, Japan. ${ }^{3}$ Institute of Materials Structure Science, High Energy Accelerator Research Organization (KEK), 1-1 Oho, Tsukuba, Ibaraki 305-0801, Japan. Correspondence and requests for materials should be addressed to K.M. (email: michishio@rs.tus.ac.jp).
} 
$\mathrm{T}$ he three-body problem with a Coulomb interaction has been the focus of attention in fundamental physics for not only classical mechanics but also quantum mechanics, since the Schrödinger equation for a three-body system has not been solved analytically, despite the proposal of a variety of approximation approaches. The $\mathrm{Ps}^{-}{ }^{-}$ion $^{1,2}$ can be regarded, from an atomic and molecular physics perspective, as an intermediate between the two extreme cases of $\mathrm{H}^{-}$(atomic-like) and $\mathrm{H}_{2}^{+}$ (molecular-like) because of its mass ratio ${ }^{3-5}$. Since the theoretical simplifications applied to atoms or molecules may often be inadequate, research on $\mathrm{Ps}^{-}$structure and dynamics can provide a stringent testing ground for the quantum mechanical three-body problem.

Theoretical studies indicate that $\mathrm{Ps}^{-}$has only a ground state $\left({ }^{1} S^{e}\right)$ where the two electrons have opposite spins, and no particlestable excited states ${ }^{6,7}$, unlike the $\mathrm{H}^{-}$ion, which has a doubly excited ${ }^{3} \mathrm{P}^{\mathrm{e}}$ state. However, quasi-bound states (resonances) have been theoretically predicted in the vicinity of the formation thresholds of Ps (for principal quantum number $n \geq 2$ ) (ref. 8), offering the expectation that experiments will reveal rich structures around the energy levels of $\mathrm{Ps}^{-}$. Although the resonance states spontaneously dissociate into Ps in the ground state or lower-lying excited state and electron in the continuum, interference between the direct detachment process and the detachment via the resonance state gives rise to characteristic structures on the cross sections near the resonance energy. The resonance of the ${ }^{1} \mathrm{P}^{\mathrm{o}}$ symmetry, which is accessible by the single-photon absorption of $\mathrm{Ps}^{-}$, has been theoretically investigated ${ }^{9-13}$. In the vicinity of the $n=2$ threshold, a strong shape resonance, in which the electron is temporarily trapped by a centrifugal barrier potential, is thought to lie above the level. Moreover, a series of Feshbach resonances, which originates from an attractive dipole potential formed by the $2 \mathrm{~S}-2 \mathrm{P}$ degeneracy of Ps $(n=2)$, is also expected to lie just below this threshold.

Historically, the existence of $\mathrm{Ps}^{-}$was predicted by Wheeler ${ }^{1}$ in 1946 and was discovered in the laboratory, using the beam-foil method, by Mills ${ }^{2}$ in 1981. Since then numerous theoretical studies have been devoted to exploring the nature of this exotic ion ${ }^{14-23}$. However, because of the extremely weak ion yield and short annihilation lifetime (479 ps), experimental investigations on $\mathrm{Ps}^{-}$ have been limited to a few measurements of its annihilation rate (ref. 24 and references therein). Recently, an efficient formation method for this ion was found where, on impacting slow positron beams onto tungsten (W) surfaces coated with sub-monolayer alkali-metal atoms, the conversion efficiency increased by double digits due to the coating $25-27$. This discovery has opened up new experimental fields for $\mathrm{Ps}^{-}$, such as its photodetachment ${ }^{28}$ and the consequent generation of an energy-tunable Ps beam ${ }^{29}$.

In this letter, we report on a study of its kind made on the laser spectroscopy of $\mathrm{Ps}^{-}$ions, generated by this efficient production scheme. We report the observation of a strong shape resonance of ${ }^{1} \mathrm{P}^{\mathrm{o}}$ near the $\mathrm{Ps}(n=2)$ formation threshold. The resonance energy and width measured are in good agreement with the result of three-body calculations.

\section{Result}

Experimental setup and procedure. A pulsed slow positron beam at the KEK-IMSS slow positron facility ${ }^{30}$ was used to synchronize the $\mathrm{Ps}^{-}$beam and a pulsed ultraviolet laser beam of sufficient photon density for the photodetachment of the short-lived $\mathrm{Ps}^{-}$ions. The positron beam, with a repetition of $50 \mathrm{~Hz}$ and pulse-width of $12 \mathrm{~ns}$ FWHM, was transported to the measurement chamber with a kinetic energy of $4.2 \mathrm{keV}$, passing through a plate with a $5 \mathrm{~mm}$ circular aperture. The beam intensity and the diameter were $4 \times 10^{3} \mathrm{e}^{+}$per pulse and $4 \mathrm{~mm}$ FWHM, respectively. As shown in Fig. 1a, it was deflected by an angle of $45^{\circ}$ along a curved magnetic field $(\sim 0.01 \mathrm{~T})$, then passed through forward and back grids biased at the same voltage of $3,400 \mathrm{~V}$ and, finally, impacted onto a $\mathrm{W}$ target coated with a 0.3 monolayer of $\mathrm{Na}$ (Supplementary Note 1). In order to maintain $\mathrm{Ps}^{-}$emission from the surface ${ }^{26}$ for the duration of the runs, the chamber was evacuated to a pressure of $1 \times 10^{-8} \mathrm{~Pa}$.

When positrons impinge onto a surface, they can lose their kinetic energies and thermalize in the bulk. Some diffuse back to the surface to form $\mathrm{Ps}^{-}$ions, and these are emitted spontaneously with a low kinetic energy governed by the Ps ${ }^{-}$ affinity $(\sim-3 \mathrm{eV})$. The formation efficiency of $\mathrm{Ps}^{-}$ions against the incident positron flux is reported to be about $2 \%$ (ref. 26). The $\mathrm{Ps}^{-}$ions formed in this setup were accelerated by the potential

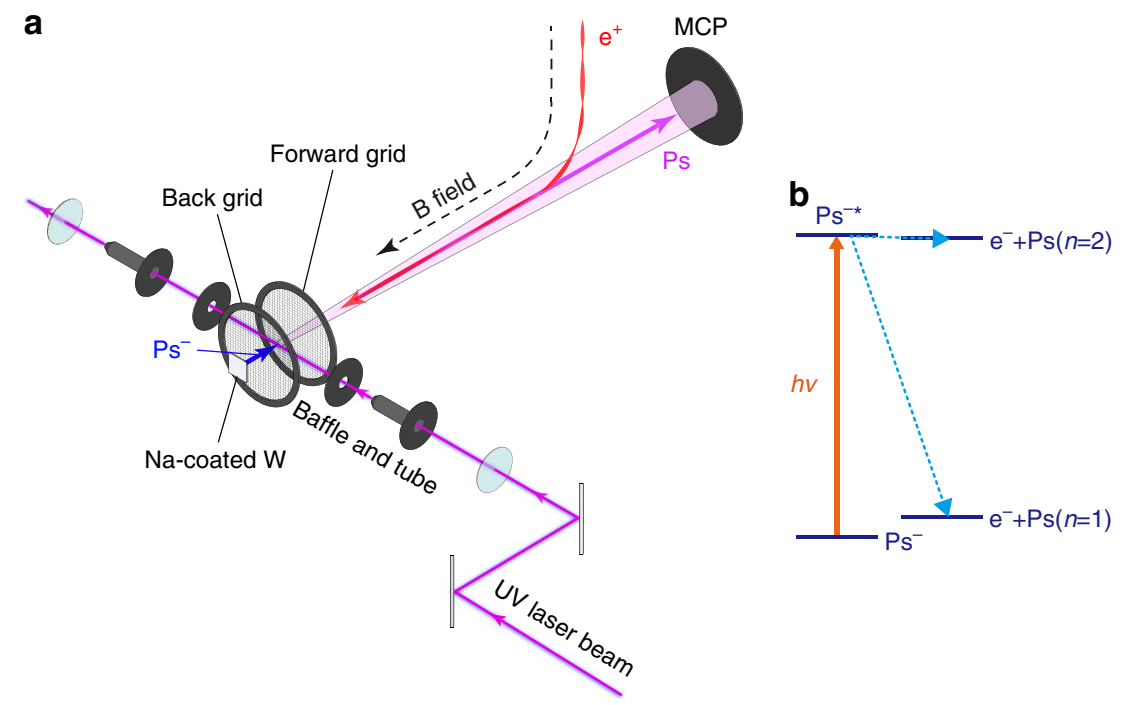

Figure 1 | Schematic diagram of the experimental setup and the energy levels of Ps ${ }^{-}$. (a) A pulsed slow positron beam is guided along a magnetic field and impacted onto a Na-coated $\mathrm{W}$ target to generate $\mathrm{Ps}^{-}$ions. The ions are accelerated by a static electric field between the target and a back grid, and are then irradiated by ultraviolet laser beam in the electric field-free region between the forward and back grids biased at the same voltage. The neutral Ps atoms formed by (resonant) photodetachment are detected by the MCP. (b) Optical transition from Ps ${ }^{-}\left({ }^{1} S^{e}\right)$ to Ps $(n=1$ or 2$)+e^{-}$continuum state via shape resonance $\left({ }^{1} \mathrm{P}^{\circ}\right)$ as indicated by $\mathrm{Ps}^{-{ }^{*}}$. 
difference, $V$, between the target and back grid. The potential of the target was varied to set the value of $V$. The ions intersected the laser beams from a tunable dye laser (see the 'Methods' section for details on the laser system) at right angle in the electric field-free region between the two grids. The effects of stray magnetic fields in the beam intersection region were considered: for a field of about $3 \times 10^{-3} \mathrm{~T}$ with a $\mathrm{Ps}^{-}$speed of $0.07 c$ $(V=3,400 \mathrm{~V})$, where $c$ is the speed of light, the effective electric field was estimated to be $6 \times 10^{2} \mathrm{~V} \mathrm{~cm}^{-1}$. Motional Starkbroadening and shift of resonance energies are small enough to be neglected at this field strength ${ }^{31,32}$.

Neutral Ps atoms formed both by the direct photodetachment process and via the resonances (Fig. 1b) were detected by a micro-channel plate (MCP), of effective diameter $42 \mathrm{~mm}$, while charged particles were removed by the curved magnetic field. The residual background was due to stray light, reflected from the laser inlet and outlet fused-silica windows coated by broadband anti-reflection coatings and annihilation $\gamma$-rays from the target. In order to reduce the MCP signal due to the stray light, baffles and cylindrical tubes with $5 \mathrm{~mm}$ diameter apertures were placed between the target and each window.

Para-Ps $(S=0)$ and ortho-Ps $(S=1)$ are formed in the $\mathrm{Ps}^{-}$photodetachment process. As for the S-states, para-Ps atoms decay with a lifetime of $125 n^{3}$ ps into two $\gamma$-rays, while ortho-Ps atoms decay with a lifetime of $142 n^{3} n$ into three $\gamma$-rays. The 2P-states, which have longer lifetimes against annihilation $(0.1-3 \mathrm{~ms})$ (refs 33,34$)$, are de-excited to $1 \mathrm{~S}$-states with a lifetime of $3.2 \mathrm{~ns}$ and these then decay according to their own annihilation lifetimes. Owing to the short flight length $(<20 \mathrm{~mm})$ of para-Ps atoms, even in the $n=2$ state, due to self-annihilation, only ortho-Ps atoms were detected by the MCP which was placed at a distance, $L$, of $0.88 \mathrm{~m}$ from the target. Although the $m=0$ states of ortho-Ps atoms are perturbed and its lifetime becomes shorter by Zeeman mixing with para-Ps atoms in a magnetic field, this effect is negligibly small, even in the Ps $(n=2)$ state at the present field strength ${ }^{35}$.

Observation. Figure 2 shows the 2D time-of-flight (TOF) spectra of the MCP signals at two different laser wavelengths for $V=3,400 \mathrm{~V}$, accumulated over $2 \times 10^{3} \mathrm{~s}$. The prompt peaks seen at time $t=0-10 \mathrm{~ns}$ are attributed mainly to the detection of stray light. Annihilation $\gamma$-rays of the positrons in the target and self-annihilation of para-Ps also contribute to these peaks. No significant signal is observed at the laser wavelength $229.7 \mathrm{~nm}$, a delayed peak is seen at $t=44 \mathrm{~ns}$ when the wavelength is tuned to $228.5 \mathrm{~nm}$. The TOF is consistent with that of Ps atoms formed by photodetachment, given by $t=L /\left(2|e| V / 3 m_{\mathrm{e}}\right)^{1 / 2}$, where $e$ and $m_{\mathrm{e}}$ are the charge and the rest mass of the electron, respectively.

The count rate of the Ps atoms, $R_{\mathrm{Ps}}$, was determined using $R_{\mathrm{Ps}}=R_{\mathrm{PL}}-R_{\mathrm{P}}-R_{\mathrm{L}}$, where $R_{\mathrm{PL}}$ and $R_{\mathrm{P}}$ are the signal rates with and without the laser irradiation, respectively, for the TOF windows of $40-50 \mathrm{~ns}(V=3,400 \mathrm{~V})$ and $62-72 \mathrm{~ns}(V=1,500 \mathrm{~V})$. $\mathrm{R}_{\mathrm{L}}$ is the background rate due to the laser irradiation. $R_{\mathrm{Ps}}$ was normalized to the average photon flux and the overlapping volume of the laser beam and the $\mathrm{Ps}^{-}$beam estimated from each spatial and temporal profile to ensure proportionality to the photodetachment cross sections (Supplementary Figs 1 and 2, and Supplementary Note 2). Figure 3 shows $R_{\mathrm{Ps}}$ measured as a function of the wavelength from $225 \mathrm{~nm}(5.51 \mathrm{eV})$ to $231 \mathrm{~nm}$ $(5.37 \mathrm{eV})$ for $V=3,400 \mathrm{~V}$ and $V=1,500 \mathrm{~V}$. Asymmetric peaks with a tail to higher photon energies were clearly observed in both cases.

\section{Discussion}

The photodetachment cross sections, $\sigma(h v)$, near resonances with energy $E_{\mathrm{r}}$ and width $\Gamma$ are often described by the Fano line profile ${ }^{36}$,

$$
\sigma(h v)=\sigma_{\mathrm{a}} \frac{(q+\epsilon)^{2}}{\left(1+\epsilon^{2}\right)}+\sigma_{\mathrm{b}}
$$

where

$$
\epsilon=\frac{h v-E_{\mathrm{r}}}{\Gamma / 2} .
$$

Here, $\sigma_{\mathrm{a}}$ and $\sigma_{\mathrm{b}}$ are the cross sections of continuum states interacting with and without the resonance state, respectively, and $q$ is the shape parameter. It has been reported that the Fano profile describes the shape resonances $\left({ }^{1} \mathrm{P}^{\circ}\right)$ of $\mathrm{H}^{-}$and $\mathrm{D}^{-}$ (refs 37,38), and was applied to molecular shape resonances ${ }^{39}$. The data obtained were fitted with this profile, as shown in Fig. 3a,b, where the fitting parameters, except for $E_{\mathrm{r}}$, were kept the same for both cases. $\sigma_{\mathrm{b}}$ was assumed to be constant. In the laboratory frame, because of the $\mathrm{Ps}^{-}$motion perpendicular to the
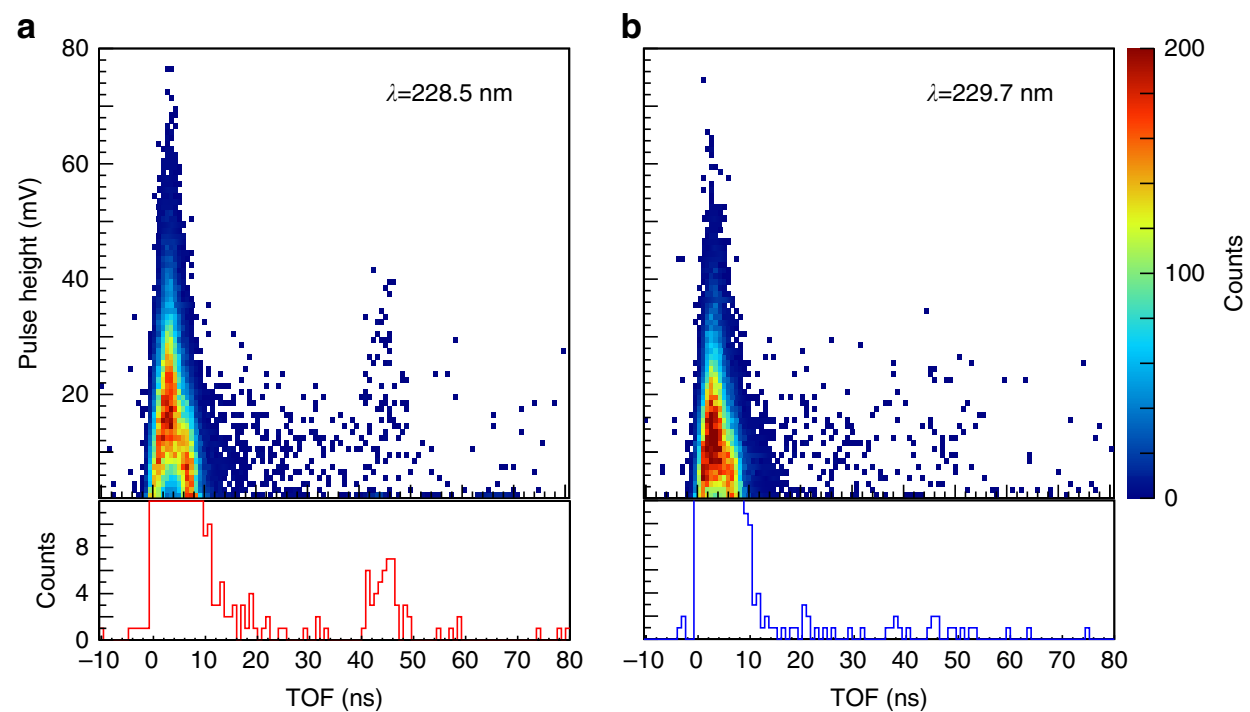

Figure 2 | 2D time-of-flight spectra of the MCP signals. The wavelengths of the laser beams were $228.5 \mathrm{~nm}$ (a) and $229.7 \mathrm{~nm}$ (b). The bottom sections are the vertical projections of the spectra with pulse height over $18 \mathrm{mV}$. When $\lambda=228.5 \mathrm{~nm}$, delayed signals from the detection of Ps atoms formed by photodetachment are observed at $t=44 \mathrm{~ns}$, while these signals are not observed for $\lambda=229.7 \mathrm{~nm}$. 
average Ps ${ }^{-}$velocity $\mathbf{v}_{z}$, transverse Doppler-broadening takes place. Accordingly, a Gaussian profile with s.d. $=1.3 \times 10^{-3} h v$, obtained in a previous measurement ${ }^{40}$, has been convoluted to the fitting profile. The values of $E_{\mathrm{r}}$ derived by the fitting were $5.4246(12) \mathrm{eV}(V=3,400 \mathrm{~V})$ and $5.4317(16) \mathrm{eV}(V=1,500 \mathrm{~V})$, where the errors represent the s.d. of the fitted values. It is clearly seen that each resonance position shifts with $V$, due to the longitudinal Doppler effect expressed as $\Delta E=-E_{\mathrm{r}}\left(v_{\mathrm{z}} / c\right)^{2} / 2$. The zero-velocity values of each $E_{\mathrm{r}}$ extracted from this formula, $5.4367(12) \mathrm{eV}(V=3,400 \mathrm{~V})$ and $5.4370(16) \mathrm{eV}(V=1,500 \mathrm{~V})$, are consistent within the s.d. Therefore the resonance energy in the rest frame of the ions was deduced to be 5.437(1) eV from the weighted arithmetic mean of these values. $E_{\mathrm{r}}$ and the other fitting parameters are listed in Table 1, along with theoretically derived values of the shape resonance by the adiabatic treatment ${ }^{9}$, the
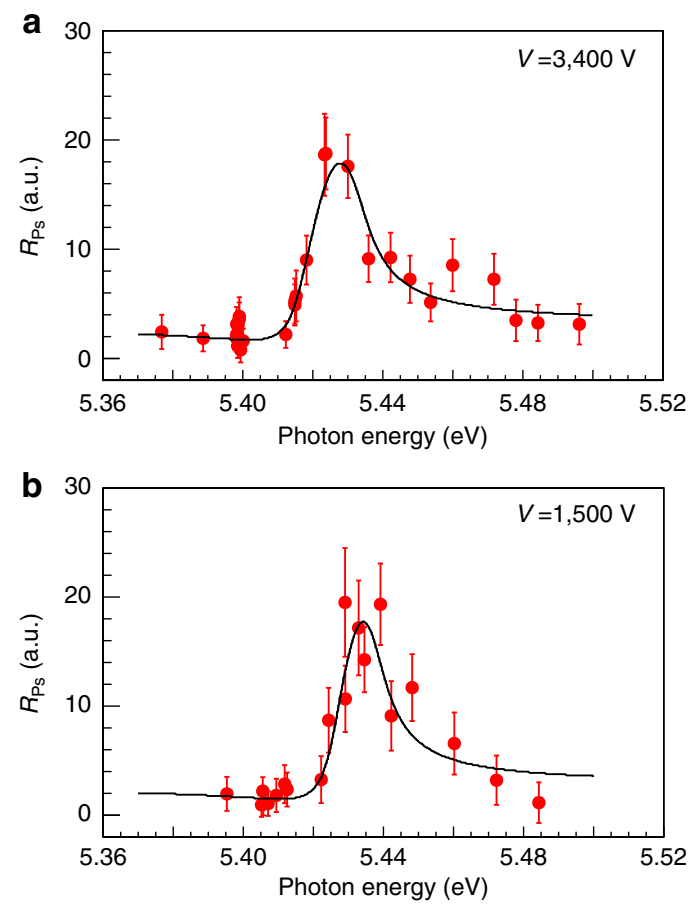

Figure 3 | Resonance profiles of $\mathbf{P s}^{-}$ions in the vicinity of the $n=2$ threshold. $R_{\mathrm{Ps}}$ plotted against photon energy for acceleration voltages of $3,400 \vee(\mathbf{a})$ and $1,500 \mathrm{~V}(\mathbf{b})$. The best fit results using a Fano profile convoluted with a Gaussian profile which represents the angular distribution of $\mathrm{Ps}^{-}$are indicated by the solid lines, where the fitting parameters, except for the resonance energy, were constrained to be the same for both sets of data $\left(\chi^{2} / v=0.66\right)$. Error bars show the standard deviation of the mean $R_{\mathrm{Ps}}$ values including the error of normalization factors. complex rotation method ${ }^{10}$ and the hyperspherical close-coupling method $^{12}$. The obtained $E_{\mathrm{r}}$ and $\Gamma$ values are in good agreement with the theoretical predictions to within meV precision. The shape parameter $q$ is also consistent with the theoretical value obtained by fitting the Fano profile to the photodetachment cross sections in the (ref. 12).

In conclusion, we have developed an experimental system for $\mathrm{Ps}^{-}$laser spectroscopy based on an efficient $\mathrm{Ps}^{-}$source. We have observed the ${ }^{1} \mathrm{P}^{\mathrm{o}}$ shape resonance in the photodetachment of $\mathrm{Ps}^{-}$ions near the $n=2$ threshold. The present experimental resolution is constrained by the Doppler width of about $7 \mathrm{meV}$ due to the $\mathrm{Ps}^{-}$motion. With a combination of the present $\mathrm{Ps}^{-}$production system and the two-photon absorption technique, in which the $\mathrm{Ps}^{-}$ions are irradiated with two counter-propagating laser beams to cancel the Doppler shift, the observation of the narrower Feshbach resonances $8,41,42$ will be feasible. This precise spectroscopy will be the next challenge for future research.

\begin{abstract}
Methods
Laser system. The light source was based on a nano-second dye laser (Sirah, Cobra-Stretch-D; dye solution: Coumarin 460) pumped by the third harmonic of a Q-switched Nd:YAG laser with a repetition of $10 \mathrm{~Hz}$. In order to extend the dye lifetime, DABCO (1, 4-diazabicyclo [2.2.2] octane) was dissolved in the dye solution at $1 \mathrm{gl}^{-1}$ (ref. 43), thereby, almost tripling the lifetime. The outputs were converted to the second harmonics by a type I BBO crystal, resulting in a wavelength range of $225-230 \mathrm{~nm}$ with a nominal linewidth of about $0.4 \mathrm{pm}$ $(9 \mu \mathrm{eV})$. The wavelength was measured using a wavelength metre (HighFinesse, WS-6). The average pulse-width of the output pulses was about $10 \mathrm{~ns}$ FWHM, and the average energy was measured to be several $10^{-4} \mathrm{~J}$ by an energy metre (Coherent, J-25MUV-193). The spatial and temporal profiles of the laser beam were continuously monitored by a beam profiler (Thorlabs, BC106-UV) and a photodiode (Thorlabs, DET10A/M), respectively. The polarization of the light was set to be parallel to the $\mathrm{Ps}^{-}$velocity vector.
\end{abstract}

Data acquisition. The waveforms of the MCP signals were recorded by a digitizer with a 10-bit resolution (National instruments, PXIe-5162). The sampling rate and the band width were $1.25 \mathrm{GS} \mathrm{s}^{-1}$ and $1.5 \mathrm{GHz}$, respectively. The characteristic properties of the laser beam (wavelength, energy, spatial profile and temporal profile) were recorded in synchronization with the digitizer. Data, with and without laser, were recorded with the repetition ratio of positrons $(50 \mathrm{~Hz})$ and laser $(10 \mathrm{~Hz})$.

Effect of positronium atoms in $\boldsymbol{n}=\mathbf{2}$ excited states. For the measurement of the resonance profile, presented in Fig. 3a,b, above the $n=2$ threshold $(5.428 \mathrm{eV}), \mathrm{Ps}$ in the $n=2$ state is formed in competition with the $n=1$ state. As for the $2^{3} \mathrm{P}$ states, they are de-excited to the $1^{3} \mathrm{~S}$ state (Lyman- $\alpha$ transition) within a lifetime of $3.2 \mathrm{~ns}$ before reaching the MCP detector, while most of the Ps in the metastable $2^{3} \mathrm{~S}$ state can reach the detector without in-flight loss since the annihilation lifetime of this state is ten times longer than that of the $1^{3} \mathrm{~S}$ state and de-excitation is forbidden. The detection efficiencies of the $2^{3} \mathrm{~S}$ state are thus 1.3 times and 1.5 times higher than those of the other states for acceleration voltages of 3,400 and 1,500 V, respectively. To evaluate this contribution, we multiplied these ratios by $2 S$ partial photodetachment cross sections calculated by the HSCC method ${ }^{12}$ and compared them with the total photodetachment cross sections with and without the multiplication. We found a shift of resonance energy of only $0.2 \mathrm{meV}$ when it was taken into account, therefore this effect was disregarded.

Table 1 | Comparison of experimental and theoretical results for the ${ }^{1} \mathbf{p}^{\mathbf{0}}$ shape resonance in the vicinity of the $n=2$ threshold.

\begin{tabular}{|c|c|c|c|c|}
\hline & \multirow{2}{*}{$\begin{array}{c}\text { Experiment } \\
\text { Present } \\
\end{array}$} & \multicolumn{3}{|c|}{ Theory } \\
\hline & & Botero et al. ${ }^{9}$ & Bhatia et al..$^{10}$ & Igarashi et al. ${ }^{12}$ \\
\hline$E_{r}(\mathrm{eV})$ & $5.437(1)$ & 5.44 & $5.438(1)$ & 5.4375 \\
\hline$\Gamma(\mathrm{eV})$ & $0.010(2)$ & 0.01 & $0.012(1)$ & 0.013 \\
\hline$q$ & $3.9(8)$ & & & $3.65^{\star}$ \\
\hline \multicolumn{5}{|c|}{$\begin{array}{l}E_{r} \text { resonance energy; } \Gamma \text {, resonance width; } q \text {, shape parameter. } \\
\text { Errors of the experimental values represent s.d. of the fitted values. } \\
\text { The resonance energy in the theory was derived with reference to a }\end{array}$} \\
\hline
\end{tabular}




\section{References}

1. Wheeler, J. A. Polyelectrons. Ann. N. Y. Acad. Sci. 48, 219-238 (1946).

2. Mills, A. P. Jr. Observation of the positronium negative ion. Phys. Rev. Lett. 46, 717-720 (1981).

3. Rost, J. M. \& Wintgen, D. Positronium negative ion: molecule or atom? Phys. Rev. Lett. 69, 2499-2502 (1992).

4. Martin, A., Richard, J.-M. \& Wu, T. T. Stability of three-unit-charge systems. Phys. Rev. A 46, 3697-3703 (1992).

5. Frolov, A. M. \& Bishop, D. M. Theory of bound states in the Coulomb three-body system with unit charges. Phys. Rev. A 45, 6236-6240 (1992).

6. Mills, A. P. Jr. Probable nonexistence of a ${ }^{3} P^{\mathrm{e}}$ metastable excited state of the positronium negative ion. Phys. Rev. A 24, 3242-3244 (1981).

7. Bhatia, A. K. \& Drachman, R. J. New calculation of the properties of the positronium ion. Phys. Rev. A 28, 2523-2525 (1983).

8. Ho, Y. K. Autoionization states of the positronium negative ion. Phys. Rev. A 19, 2347-2352 (1979).

9. Botero, J. \& Greene, C. H. Resonant photodetachment of the positronium negative ion. Phys. Rev. Lett. 56, 1366-1369 (1986).

10. Bhatia, A. K. \& Ho, Y. K. Complex-coordinate calculation of ${ }^{1,3} P$ resonances in $\mathrm{Ps}^{-}$using Hylleraas functions. Phys. Rev. A 42, 1119-1122 (1990).

11. Ho, Y. K. \& Bhatia, A. K. P-wave shape resonances in positronium ions. Phys. Rev. A 47, 1497-1499 (1993).

12. Igarashi, A., Shimamura, I. \& Toshima, N. Photodetachment cross sections of the positronium negative ion. New J. Phys. 2, 17 (2000).

13. Igarashi, A. \& Shimamura, I. Time-delay matrix analysis of resonances: application to the positronium negative ion. J. Phys. B: At. Mol. Phys. 37, 4221-4237 (2004).

14. Kolos, W., Roothaan, C. C. \& Sack, R. A. Ground state of systems of three particles with Coulomb interaction. Rev. Mod. Phys. 32, 178-179 (1960).

15. Frost, A. A., Inokuti, M. \& Lowe, J. P. Approximate series solutions of nonseparable Schrödinger equations. II. general three-particle system with Coulomb interaction. J. Chem. Phys. 41, 482-489 (1964).

16. Bhatia, A. K. \& Drachman, R. J. Photodetachment of the positronium negative ion. Phys. Rev. A 32, 3745-3747 (1985).

17. Ward, S. J., Humberston, J. W. \& McDowell, M. R. C. Elastic scattering of electrons (or positrons) from positronium and the photodetachment of the positronium negative ion. J. Phys. B: At. Mol. Phys. 20, 127-149 (1987).

18. Ho, Y. K. Variational calculation of ground-state energy of positronium negative ions. Phys. Rev. A 48, 4780-4783 (1993).

19. Ivanov, I. A. \& Ho, Y. K. Supermultiplet structure of the doubly excited positronium negative ion. Phys. Rev. A 61, 032501 (2000).

20. Frolov, A. Positron annihilation in the positronium negative ion $\mathrm{Ps}^{-}$. Phys. Lett. A 342, 430-438 (2005).

21. Drake, G. W. F. \& Grigoreseu, M. Binding energy of the positronium negative ion: relativistic and QED energy shifts. J. Phys. B 38, 3377-3393 (2005).

22. Puchalski, M., Czarnecki, A. \& Karshenboim, S. G. Positronium-ion decay. Phys. Rev. Lett. 99, 203401 (2007).

23. Frolov, A. M. Highly accurate evaluation of the singular properties for the positronium and hydrogen negative ions. J. Phys. A: Math. Theor. 40, 6175-6181 (2007).

24. Ceeh, H. et al. Precision measurement of the decay rate of the negative positronium ion Ps ${ }^{-}$. Phys. Rev. A 84, 062508 (2011).

25. Nagashima, Y., Hakodate, T., Miyamoto, A. \& Michishio, K. Efficient emission of positronium negative ions from Cs deposited W(100) surfaces. New J. Phys. 10, 123029 (2008)

26. Terabe, H., Michishio, K., Tachibana, T. \& Nagashima, Y. Durable emission of positronium negative ions from $\mathrm{Na}$ - and K-coated W(100) surfaces. New J. Phys. 14, 015003 (2012).

27. Nagashima, Y. Experiments on positronium negative ions. Phys. Rep. 545, 95-123 (2014)

28. Michishio, K. et al. Photodetachment of positronium negative ions. Phys. Rev. Lett. 106, 153401 (2011).

29. Michishio, K. et al. An energy-tunable positronium beam produced using the photodetachment of the positronium negative ion. Appl. Phys. Lett. 100, 254102 (2012)

30. Wada, K. et al. New experiment stations at KEK slow positron facility. J. Phys. Conf. Ser. 443, 012082 (2013)
31. Ho, Y. K. \& Ivanov, I. A. dc Stark effect for doubly excited Feshbach resonance states of the positronium negative ion below the $N=2$ threshold of a positronium atom. Phys. Rev. A 63, 062503 (2001).

32. Comtet, G. et al. Stability of the ${ }^{1} P^{\mathrm{o}}$ shape resonance in $\mathrm{H}^{-}$in moderate electric fields. Phys. Rev. A 35, 1547-1554 (1987).

33. Alekseev, A. I. Two-photon annihilation of positronium in the P-state. Sov. Phys. JETP 34, 826-830 (1958).

34. Alekseev, A. I. Three-photon annihilation of positronium in the P-state. Sov. Phys. JETP 36, 1312-1315 (1959).

35. Curry, S. M. Combined Zeeman and motional Stark effects in the first excited state of positronium. Phys. Rev. A 7, 447-450 (1973).

36. Fano, U. Effects of configuration interaction on intensities and phase shifts Phys. Rev. 124, 1866-1878 (1961).

37. Halka, M. et al. Branching ratio of the $\mathrm{H}^{-}(n=2)$ shape resonance. Phys. Rev. A 46, 6942-6948 (1992).

38. Balling, P. et al. Doppler tuning vuv spectroscopy of $\mathrm{D}^{-}$over an extended photon-energy range around the $n=2$ threshold. Phys. Rev. A 76, 044701 (2007).

39. Gil, T. J., Winstead, C. L., Sheehy, J. A. \& Farren, R. E. New theoretical perspectives on molecular shape resonances: Feshbach-Fano methods for Mulliken orbital analysis of photoionization continua. Phys. Scr. T31, 179-188 (1990).

40. Michishio, K. et al. Profiles of a positronium beam produced using the photodetachment of positronium negative ions. Nucl. Inst. Methods A 785, 5-8 (2015).

41. Bhatia, A. K. \& Ho, Y. K. Complex-coordinate calculations of doubly excited ${ }^{1,3} D^{\mathrm{e}}$ resonant states of Ps ${ }^{-}$. Phys. Rev. A 48, 264-267 (1993).

42. Igarashi, A. Calculation of two-photon detachment cross section of the positronium negative ion. J. Phys. B 45, 245201 (2012).

43. Trebra, R. V. \& Koch, T. H. DABCO stabilization of coumarin dye lasers. Chem. Phys. Lett. 93, 315-317 (1982).

\section{Acknowledgements}

We thank Akinori Igarashi for helpful discussion and providing calculated values. We also thank the staff of the Photon Factory and the Accelerator Laboratory of KEK for their support. This work was conducted under the approval of the Photon Factory Program Advisory Committee (Proposal No. 2013S2-005). It was supported by JSPS KAKENHI Grant Numbers 24221006 and 25887046 . T.K. is financially supported by MATSUO FOUNDATION.

\section{Author contributions}

K.M. designed the apparatus and carried out the measurements with S.K. and T.K. The laser system was developed by T.K. The data was analysed by K.M. and S.K. K.W., I.M., A.Y. and T.H. provided the support on the slow positron beam line. Y.N. and T.A proposed and supervised the experiment. The manuscript was prepared by K.M., S.K., T.A. and Y.N. and then discussed with all authors.

\section{Additional information}

Supplementary Information accompanies this paper at http://www.nature.com/ naturecommunications

Competing financial interests: The authors declare no competing financial interests.

Reprints and permission information is available online at http://npg.nature.com/ reprintsandpermissions/

How to cite this article: Michishio, K. et al. Observation of a shape resonance of the positronium negative ion. Nat. Commun. 7:11060 doi: 10.1038/ncomms11060 (2016)

This work is licensed under a Creative Commons Attribution 4.0 International License. The images or other third party material in this article are included in the article's Creative Commons license, unless indicated otherwise in the credit line; if the material is not included under the Creative Commons license, users will need to obtain permission from the license holder to reproduce the material. To view a copy of this license, visit http://creativecommons.org/licenses/by/4.0/ 